\title{
The prognostic performance of Sepsis-3 and SIRS criteria for patients with urolithiasis-associated sepsis transferred to ICU following surgical interventions
}

\author{
BOWEN SHI $^{1 *}$, FEI SHI $^{2 *}, \mathrm{KE} \mathrm{XU}^{3^{*}}$, LIUHUI SHI $^{2}$, HAIXIAO TANG $^{1}$, \\ NING WANG ${ }^{1}$, YANYUAN WU ${ }^{1}$, JUN GU $^{1}$, JIE DING $^{1}$ and YUNTENG HUANG ${ }^{1}$ \\ ${ }^{1}$ Department of Urology, School of Medicine, Xin Hua Hospital Affiliated to Shanghai Jiao Tong University, \\ Shanghai 200092; ${ }^{2}$ Department of Urology, Civil Aviation Hospital, Shanghai 200000; ${ }^{3}$ Department of Urology, \\ Changshu Hospital Affiliated to Nanjing University of Chinese Medicine, Changshu, Jiangsu 215500, P.R. China
}

Received February 2, 2019; Accepted July 25, 2019

DOI: $10.3892 /$ etm.2019.8057

\begin{abstract}
The aim of the present study was to validate the prognostic effectiveness of Sepsis-3 criteria, including sequential organ failure assessment (SOFA) and quick SOFA (qSOFA), with systemic inflammatory response syndrome (SIRS) criteria among patients with urolithiasis associated sepsis that were transferred to intensive care unit (ICU) facilities following surgical interventions. To achieve this, the records of all patients transferred to ICU following surgical interventions with urolithiasis-associated sepsis between January 2010 to July 2017 at Xin Hua Hospital Affiliated to Shanghai Jiao Tong University were retrospectively reviewed. A total of 107 patients were enrolled. The prognostic performances of SOFA, qSOFA and SIRS for predicting in-hospital mortality (sepsis-related mortality during patients' hospitalizations) or prolonged length of ICU stay ( $>3$ days) were compared using the area under the receiver operating characteristic curve (AUROC) and Z statistic values. The results revealed that the overall in-hospital mortality rate was $8.4 \%$ and the percentage of in-hospital mortality or prolonged length of ICU stay (>3 days) was $72.0 \%$ among the 107 patients. The favorable outcome group exhibited significantly decreased white blood cell counts, and levels of $\mathrm{C}$-reactive protein and procalcitonin and increased systolic blood pressure and mean arterial pressure. The AUROC of qSOFA, SIRS and SOFA were $0.615,0.625$ and 0.860 , respectively. SOFA was significantly
\end{abstract}

Correspondence to: Dr Jie Ding or Dr Yunteng Huang, Department of Urology, School of Medicine, Xin Hua Hospital Affiliated to Shanghai Jiao Tong University, 1665 Kongjiang Road, Shanghai 200092, P.R. China

E-mail: jiaodaaniu@hotmail.com

E-mail: huangyunteng@xinhuamed.com.cn

*Contributed equally

Key words: quick sequential organ failure assessment, sepsis, systemic inflammatory response syndrome, urolithiasis more effective at predicting adverse outcomes when compared with SIRS and qSOFA criteria. Following adjustments for patient age and comorbidities, the AUROC values of qSOFA, SIRS and SOFA were $0.713,0.722$ and 0.940 . In conclusion, the results of the present study indicate that the prognostic performance of SOFA for predicting in-hospital mortality or prolonged ICU stay among patients with urolithiasis-associated sepsis following surgical interventions was significantly improved when compared with qSOFA or SIRS criteria. Based on these results it is recommended that urologists use the SOFA score for patients with urolithiasis-associated sepsis.

\section{Introduction}

Sepsis is a major health problem and a leading cause of mortality and critical illness worldwide (1-3). It presents a syndrome of physiological, pathological and biochemical abnormalities induced by infection (3). The updated Sepsis-3 criteria defines sepsis as life-threatening organ dysfunction caused by a dysregulated host response to infection (3-5). For clinical operationalization, an increase in sequential organ failure assessment (SOFA) score of $\geq 2$ points may indicate organ dysfunction and an associated $>10 \%$ change of in-hospital mortality (3). Urosepsis is defined as sepsis caused by a urogenital tract infection and it may be caused by urinary tract obstruction or complication following surgical interventions (6-8). Urolithiasis-associated sepsis, caused by obstructive upper ureteral calculi or postoperative complications following percutaneous nephrolithotomy (PCNL), ureteroscopic lithotripsy (URL) and laparoscopic lithotomy, is a severe global problem in the field of urology (9-13). To the best of our knowledge, there is no consensus on whether the Sepsis-3 criteria are an effective prognostic tool for patients with urolithiasis-associated sepsis.

A number of studies have evaluated the effectiveness of Sepsis-3 criteria for predicting in-hospital mortality among patients with suspected infection that are admitted to the emergency department (ED) or intensive care unit (ICU) (14-19). Studies have demonstrated that SOFA scores are superior for predicting mortality when compared to 
systemic inflammatory response syndrome (SIRS) and quick SOFA (qSOFA) criteria $(15,18,19)$. However, there may be unique differences between urosepsis and sepsis caused by other factors, particularly urolithiasis-associated sepsis that develops following surgical interventions $(6-8,20-22)$. In addition, there may be differences in the application of Sepsis-3 criteria among different countries and at different levels of health care. Furthermore, it is clear that urologists are unfamiliar with the new definition of sepsis when compared with clinicians working in the ED or ICU.

The aim of the present study was to compare the effectiveness of Sepsis-3 (SOFA and qSOFA) and SIRS criteria for predicting in-hospital mortality or prolonged length of ICU stay ( $>3$ days) among patients with urolithiasis-associated sepsis that were transferred to the ICU following surgical interventions.

\section{Materials and methods}

Patients and methods. In the present study, the records of all patients who had been transferred to ICU following surgical interventions with urolithiasis-associated sepsis in our institution (Xin Hua Hospital Affiliated to Shanghai Jiao Tong University) from January 2010 to July 2017 were retrospectively reviewed. Due to the lack of application of SOFA criteria in the urology department, certain patients were not included. The study population consisted of patients with urosepsis caused by obstructive upper ureteral or renal calculi who were transferred to ICU following emergency surgical decompression (ureteroscopic double J stenting), or patients with postoperative sepsis following PCNL or URL who were then transferred to ICU. Due to the lack of critical life-support device and $24 \mathrm{~h}$ care available, patients with suspicious sepsis in all surgical departments should be transferred to the specialized surgical intensive care unit (SICU) in Xin Hua Hospital Affiliated to Shanghai Jiao Tong University. For convenience, the definitions of ICU and SICU are used interchangeably in the present study. The Sepsis-3 and SIRS criteria scores were calculated based on the results of initial laboratory tests and physical examination performed upon admission to the SICU. Altered mental status, within the qSOFA criteria, was defined using the Glasgow Coma Scale, with a score of $<15$, or any clinical signs, determined by clinicians. Patients with no complete previous medical record of chronic renal, liver or respiratory dysfunction, were assigned a baseline SOFA score of 0 (15). In the present study, in-hospital mortality was defined as sepsis-associated mortality at any time during patient hospitalizations. In-hospital mortality and prolonged length of ICU stay ( $>3$ days) were considered as adverse outcomes, which the Sepsis-3 and SIRS criteria may predict.

All patients were examined preoperatively with urinary ultrasonography or/and computed tomography in the ED or urology department to evaluate the existence of urolithiasis. Consistent with previous studies, evidence of infection was identified through the analysis of radiological studies, microbiological data or clinical symptoms (14). The diagnosis of urosepsis was conducted by the patient's primary surgeon or the on-duty senior surgeon according to SIRS criteria (January 2010 to March 2016) and SOFA scores (April 2016 to July 2017). In certain circumstances, the decision was made based on personal experiences of the clinicians, or conclusions were drawn from a consensus of several urology experts. Exclusion criteria were as follows: Age, $<18$ years old; patients who were receiving regular chemotherapy or immunosuppressant therapy; patients with unidentifiable sources of infection from the urinary tract; patients with multiple system infections without an identifiable primary source; patients who did not undergo any surgical interventions prior to transfer to the SICU; and patients with incomplete retrospective data for measuring Sepsis-3 and SIRS criteria. The flow diagram of patient enrollment is presented in Fig. 1.

Surgical procedure. The methods used to perform PCNL, URL and emergency ureteroscopic double J stenting procedures were analogous with other previously described methods $(11,12,23)$. Patients with obstructive urolithiasis and suspected urosepsis, as diagnosed by the emergency physicians, were transferred to the urology department. Following assessment of their condition, patients underwent emergency ureteroscopic double J stenting under local, spinal or general anesthesia and were then transferred to SICU. Conversely, following routine PCNL or URL, patients with suspected postoperative urosepsis were directly transferred to SICU. Generally, all patients were treated immediately with appropriate broad-spectrum intravenous antibiotics and resuscitation with intravenous fluids when the occurrence or symptoms of sepsis were confirmed. All procedures were performed by experienced physicians, while emergency ureteroscopic double J stenting was occasionally performed by younger residents.

Ethics approval and consent to participate. The Ethics Committee of Xin Hua Hospital Affiliated to Shanghai Jiao Tong University School of Medicine approved this retrospective study and permitted the waiver of written informed consent from patients (approval no., XHEC-D-2018-074). The study protocol was performed in accordance with the Declaration of Helsinki.

Statistical analysis. All analyses were performed using SPSS $^{\circledR}$ (v.19.0; SPSS Software, Inc.), R (v.3.5.1) with RStudio (v.1.1.456) and MedCalc (v.18.2.1). Data were presented as the mean \pm standard deviation or medians with interquartile ranges for continuous variables, numbers and proportions for categorical variables. Comparisons between groups were conducted using $\chi^{2}$ tests for proportions and unpaired Student's $\mathrm{t}$-tests for normally distributed data. All P-value analyses were two-sided and $\mathrm{P}<0.05$ was considered to indicate a statistically significant difference. The predictive performance of SOFA, qSOFA and SIRS for the primary outcome (in-hospital mortality and prolonged length of ICU stay) was assessed. Prognostic discrimination was determined by comparing the area under the receiver operating characteristic curve (AUROC) for each criterion (SOFA, qSOFA and SIRS) individually (14) as described previously using the $\mathrm{Z}$ statistic. Furthermore, AUROC were adjusted for patient age and comorbidities using binary logistic regression. The covariables used included: Age; hypertension; cardiovascular disease; chronic obstructive pulmonary disease; diabetes mellitus; cerebrovascular disease; chronic kidney disease; history of malignancy; SIRS; SOFA; and qSOFA. The categorical covariates included: 
Patients with clinically diagnosed urolithiasis associated sepsis and transferred to SICU

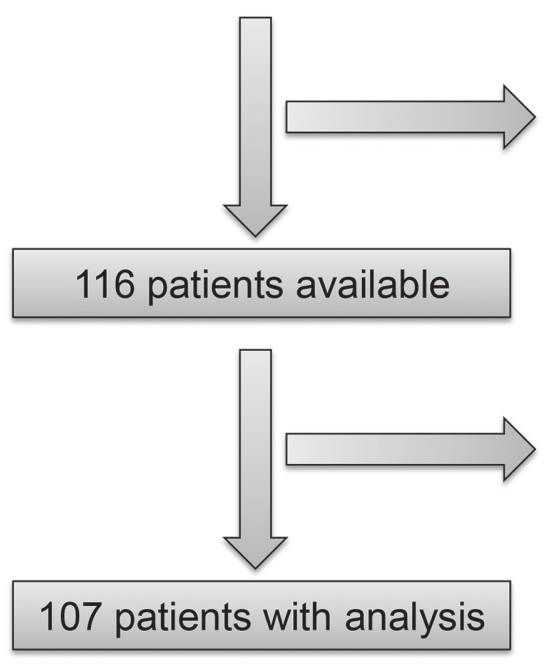

\begin{tabular}{|l|}
\hline 12 patients excluded \\
5 without surgical interventions \\
2 younger than 18 \\
1 suspect existence of urolithiasis \\
2 receiving chemotherapy \\
2 receiving immunosuppressant \\
therapy
\end{tabular}

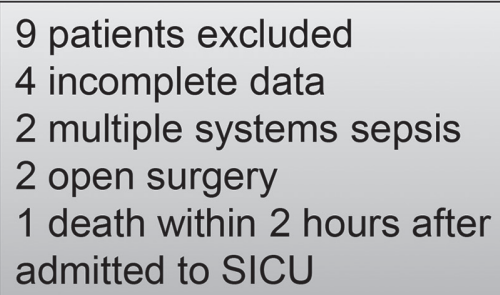

Figure 1. Flow diagram of patient enrollment. SICU, surgical intensive care unit.

Hypertension; cardiovascular disease; chronic obstructive pulmonary disease; diabetes mellitus; cerebrovascular disease; chronic kidney disease; history of malignancy; SIRS; SOFA; and qSOFA.

\section{Results}

Patient characteristics and outcomes. A total of 107 patients (72 females and 35 males) enrolled in the study cohort and their results were statistically analyzed. These included 63 patients that had undergone emergency ureteroscopic double-J stenting, 20 patients following PCNL and 24 patients following URL. Their detailed baseline characteristics are summarized in Table I. The overall in-hospital mortality rate was $8.4 \%$ (9/107), and the percentage of in-hospital mortality or prolonged length of ICU stay (>3 days) was $72.0 \%(77 / 107)$. The median age was 66 (range, 54-77) years and $32.7 \%$ were male. The primary comorbidities included: Hypertension (46.7\%); cardiovascular disease $(14.0 \%)$; chronic obstructive pulmonary disease (13.1\%); diabetes mellitus (22.4\%); cerebrovascular disease (15.0\%); chronic kidney disease (11.2\%); gout $(6.5 \%)$; and history of malignancy (7.5\%). The median of qSOFA score was 2 (range, 1-3) and $84.1 \%$ patients exhibited a qSOFA score of $\geq 2$. The median of SOFA score was 5 (range, $0-15$ ) and $80.14 \%$ patients exhibited an increase in SOFA score of $\geq 2$. The median of patients' SIRS criteria was 4 (range, 2-4) and all patients exhibited $\geq 2$ SIRS criteria. The distributions of each score (SOFA, qSOFA and SIRS) are presented in Fig. 2A-C.

Comparisons between favorable $(n=30)$ and adverse outcome groups $(n=77)$. No difference in the number of patients that had undergone each type of surgical procedure in the favorable and adverse outcome groups was observed (PCNL, 20 vs. $18.2 \%$; URL, 20 vs. $23.4 \%$; DJ stent, 60 vs. $58.4 \%$ ).
No significant differences in age and comorbidities were observed between the two groups. However, the percentage of males was significantly increased in the favorable outcome group. The favorable outcome group also exhibited significantly decreased white blood cell (WBC) counts (13.95 vs. $19.60 \times 10^{9} / 1, \mathrm{P}<0.001$ ), and levels of C-reactive protein (48 vs. $160 \mathrm{mg} / \mathrm{l}, \mathrm{P}=0.002)$ and procalcitonin $(2.18 \mathrm{vs} .29 .60 \mathrm{ng} / \mathrm{ml}$, $\mathrm{P}<0.001)$. Systolic blood pressure $(84.5$ vs. $76 \mathrm{mmHg}, \mathrm{P}<0.001)$ and mean arterial pressure $(63.3$ vs. $58.3 \mathrm{mmHg}, \mathrm{P}<0.001)$ were significantly decreased in the adverse outcome group. The level of patients with altered mental state was significantly increased in the adverse outcome group. The SOFA, qSOFA scores and SIRS criteria were significantly increased in adverse outcome groups. In addition, the percentage of patients with SOFA score $\geq 2$ points and qSOFA $\geq 2$ points was significantly increased in adverse outcome group. The results for all parameters are summarized in Table I.

Prognostic performance. For the prediction of adverse outcomes (in-hospital mortality or length of ICU stay $>3$ days), the AUROC values of qSOFA, SIRS and SOFA were 0.615 [95\% confidence interval (CI), 0.516-0.707], 0.625 (95\% CI, $0.526-0.716$ ) and 0.860 (95\% CI, 0.780-0.920), respectively. SOFA scores were significantly improved compared with SIRS (0.860 vs. $0.625, \mathrm{P}<0.0001)$ and qSOFA (0.860 vs. 0.615 , $\mathrm{P}=0.0002)$ for the prediction of adverse outcome. There was no significant difference between SIRS and qSOFA $(0.625$ vs. $0.615, \mathrm{P}=0.887$ ). SOFA score $\geq 2$ points presented high sensitivity at $97.40 \%$ and moderate specificity at $63.33 \%$ for prediction. The cutoff of SIRS was set as $>2$ criteria (normally $\geq 2$ ). The detailed results are summarized in Tables II, III, IV and Fig. 3.

Adjusted AUROC. The AUROC values of the models used to predict adverse outcomes were adjusted for patient age and 
Table I. Baseline characteristics.

\begin{tabular}{|c|c|c|c|c|}
\hline Variable & All patients $(\mathrm{n}=107)$ & $\begin{array}{l}\text { Favorable outcome, } \\
\text { SICU stay } \leq 3 \text { days } \\
\quad(n=30)\end{array}$ & $\begin{array}{l}\text { Adverse outcome, } \\
\text { in-hospital mortality } \\
\text { or SICU stay } \\
>3 \text { days }(\mathrm{n}=77)\end{array}$ & P-value \\
\hline Age, years, median (IQR), & $66(54-77)$ & $64(54-73)$ & $67(54-80)$ & 0.19 \\
\hline Sex, male, $\%$ & $35(32.7)$ & $21(70.0)$ & $14(18.2)$ & $<0.001$ \\
\hline \multicolumn{5}{|l|}{ Surgical procedures, $\mathrm{n}(\%)$} \\
\hline PCNL & $20(18.7)$ & $6(20.0)$ & $14(18.2)$ & 0.83 \\
\hline URL & $24(22.4)$ & $6(20.0)$ & $18(23.4)$ & 0.71 \\
\hline DJ stent & $63(58.9)$ & $18(60.0)$ & $45(58.4)$ & 0.88 \\
\hline \multicolumn{5}{|l|}{ Comorbidities, n (\%) } \\
\hline Hypertension & $50(46.7)$ & $12(40.0)$ & $38(49.4)$ & 0.384 \\
\hline Cardiovascular disease & $15(14.0)$ & $4(13.3)$ & $11(14.3)$ & 1.0 \\
\hline Chronic obstructive pulmonary disease & $14(13.1)$ & $4(13.3)$ & $10(13.0)$ & 1.0 \\
\hline Diabetes mellitus & $24(22.4)$ & $5(16.7)$ & $19(24.7)$ & 0.372 \\
\hline Cerebrovascular disease & $16(15.0)$ & $4(13.3)$ & $12(15.6)$ & 1.0 \\
\hline Chronic kidney disease & $12(11.2)$ & $5(16.7)$ & $7(9.1)$ & 0.265 \\
\hline Gout & $7(6.5)$ & $3(10.0)$ & $4(5.2)$ & 0.64 \\
\hline History of malignancy & $8(7.5)$ & $2(6.7)$ & $6(7.8)$ & 1.0 \\
\hline \multicolumn{5}{|l|}{ Laboratory results, median (IQR) } \\
\hline $\mathrm{WBC}, \times 10^{9} / 1$ & $16.21(11.30-23.54)$ & $13.95(7.07-15.51)$ & $19.60(13.40-24.80)$ & $<0.001$ \\
\hline C-reactive protein, $\mathrm{mg} / \mathrm{l}$ & $118(45-160)$ & $48(12-160)$ & $160(65-160)$ & 0.002 \\
\hline $\mathrm{PCT}, \mathrm{ng} / \mathrm{ml}$ & $17.91(3.43-96.23)$ & $2.18(0.23-7.77)$ & $29.60(13.82-100.00)$ & $<0.001$ \\
\hline \multicolumn{5}{|l|}{ Vital signs } \\
\hline Temperature, ${ }^{\circ} \mathrm{C}$ & $38.5(38.0-38.9)$ & $38.65(38.0-39.0)$ & $38.5(38-38.9)$ & 0.32 \\
\hline Systolic blood pressure, $\mathrm{mmHg}$ & $78(72-83)$ & $84.5(79-90)$ & $76(71-81)$ & $<0.001$ \\
\hline Mean arterial pressure, $\mathrm{mmHg}$ & $59.3(56-63)$ & $63.3(60.0-66.3)$ & $58.3(55.7-60.3)$ & $<0.001$ \\
\hline Respiratory rate,$/ \mathrm{min}$ & $27(23-32)$ & $26(22-32)$ & $27(23-31)$ & 0.89 \\
\hline Heart rate, $/ \mathrm{min}$ & $114(103-125)$ & $116.5(105-127)$ & $113(103-125)$ & 0.55 \\
\hline Altered mental status, n (\%) & $11(10.3)$ & $0(0)$ & $11(14.3)$ & 0.032 \\
\hline \multicolumn{5}{|l|}{ Evaluation criteria } \\
\hline SOFA & $5(2-8)$ & $1(1-3)$ & $6(4-9)$ & $<0.001$ \\
\hline qSOFA & $2(2-2)$ & $2(1-2)$ & $2(2-2)$ & 0.01 \\
\hline SIRS & $4(3-4)$ & $3.5(3-4)$ & $4(4-4)$ & 0.04 \\
\hline $\mathrm{SOFA} \geq 2, \mathrm{n}(\%)$ & $86(80.4)$ & $11(36.7)$ & $75(97.4)$ & $<0.001$ \\
\hline $\mathrm{qSOFA} \geq 2, \mathrm{n}(\%)$ & $90(84.1)$ & $21(70)$ & $69(89.6)$ & 0.034 \\
\hline SIRS $\geq 2, \mathrm{n}(\%)$ & $107(100)$ & $30(100)$ & 77 (100) & NA \\
\hline
\end{tabular}

PCNL, percutaneous nephrolithotomy; URL, ureteroscopic lithotripsy; WBC, white blood count; PCT, procalcitonin; DJ, double J; SOFA, sequential (sepsis-related) organ failure assessment; qSOFA, quick sequential (sepsis-related) organ failure assessment; SIRS, systemic inflammatory response syndrome; NA, not available.

comorbidities using binary logistic regression (Tables SI-III). The adjusted AUROC values of qSOFA, SIRS and SOFA were 0.713 (95\% CI, 0.618-0.797), 0.722 (95\% CI, 0.627-0.804) and 0.940 (95\% CI, 0.877-0.977), respectively. Comparing the prognostic value of each criteria using the adjusted AUROC values yielded similar results as the comparisons using the crude AUROC values. SOFA was significantly improved compared with SIRS and qSOFA for the prediction of adverse outcomes, and no significant difference between SIRS and qSOFA was observed. The detailed results are described in Tables II, V and Fig. 4.

\section{Discussion}

To the best of our knowledge, the present study was the first to validate and compare the prognostic performance of Sepsis-3 and SIRS criteria in patients with urolithiasis-associated sepsis. However, it remains unknown whether Sepsis-3 and SIRS criteria are good prognostic tools for in-hospital mortality or prolonged ICU stay in these specific patients, particularly those that have undergone modern urologic endoscopic lithotripsy procedures. Considering the size of the study population, 

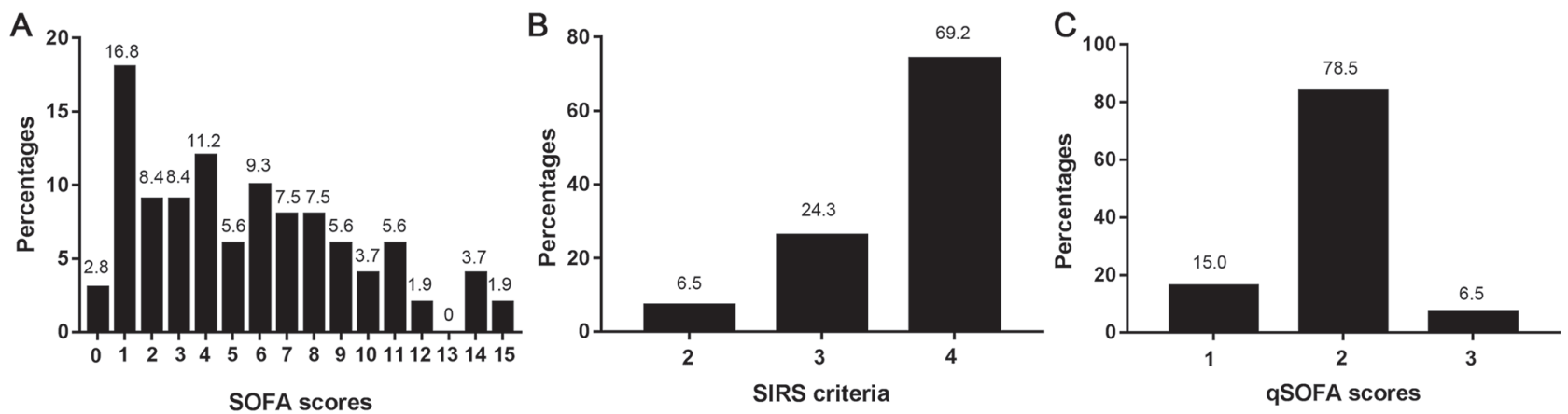

Figure 2. Diagnostic criteria score distributions. (A) SOFA score. (B) SIRS criteria. (C) qSOFA score. SOFA, sequential (sepsis-related) organ failure assessment; SIRS, systemic inflammatory response syndrome; qSOFA, quick sequential (sepsis-related) organ failure assessment.

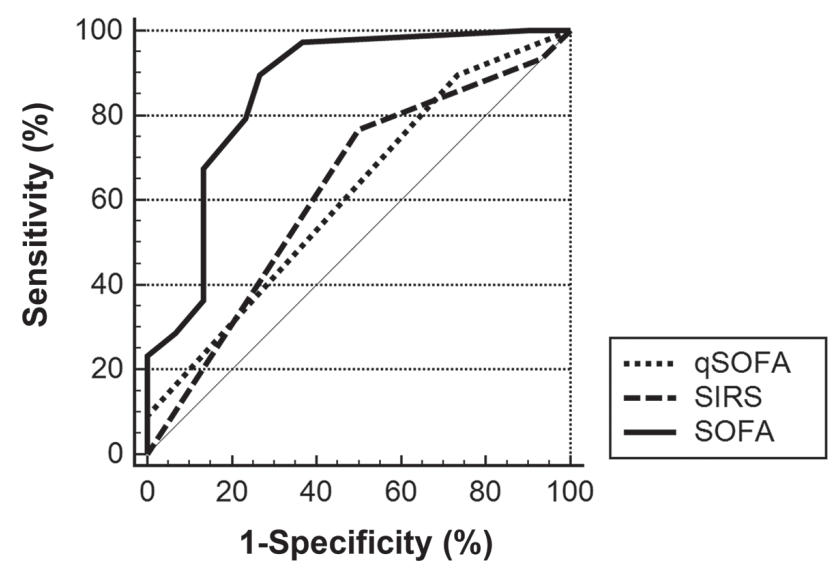

Figure 3. Comparison of prognostic performances with crude area under the receiver operating characteristic curve values. SOFA, sequential (sepsis-related) organ failure assessment; SIRS, systemic inflammatory response syndrome; qSOFA, quick sequential (sepsis-related) organ failure assessment.

in-hospital mortality or prolonged ICU stays ( $>3$ days) were set as the primary outcomes, which may be considered as secondary outcomes for other large-scale studies. SOFA score, SIRS criteria and qSOFA score have been validated and compared in several previous studies involving patients with suspected infection admitted to ICU or ED $(14,15)$ for their prognostic accuracy. These large-scale prospective studies demonstrated the advantages of the Sepsis-3 criteria compared with SIRS. However, a previous study has indicated that SIRS was associated with organ dysfunction and mortality, so it would be premature to entirely discard this criteria (19). qSOFA criteria demonstrated high specificity in a prospective ED database (19); however, poor sensitivity may limit its utility as a bedside screening method. Several statements were provided by the Surviving Sepsis Guideline panel on early management and resuscitation for sepsis or septic shock patients in a recent update (24). However, this study population was primarily characterized using the Sepsis-1 guidelines (24). This previous definition of sepsis included: Sepsis; severe sepsis; and septic shock (24). Therefore, a number of studies have established these guidelines without re-unification of the new definition of sepsis and septic shock (24). It takes time to validate, accept and incorporate novel definitions into general practice among clinicians and policymakers. Furthermore, previous studies may focus on the universal prognostic tool for all sepsis derived from different systems. For the purposes of the present study, it was considered more important to verify the prognostic accuracy of patients with urolithiasis-associated sepsis only. In addition, the aim of the present study was to evaluate the effectiveness of Sepsis-3 and SIRS criteria among patients following surgical interventions. It is likely that the composition of these patients may differ from ordinary patients in the ED or ICU.

In sepsis, disease-specific severity scores may have excellent prognostic performance, as the classifications were derived from databases of these specific diseases (16). It is therefore apparent that qSOFA, SOFA and SIRS criteria, which were developed for general infectious diseases (16), may differ from urosepsis or specific urolithiasis-associated sepsis severity classifications. Unfortunately, there were no valid and specific severity classifications used for patients with urolithiasis-associated sepsis in the present study. However, a uniform standard or classification of different infectious diseases may be a convenient tool for clinicians to utilize, particularly outside of ICU or ED. Urologists may be unfamiliar with the new definition and scale scores of Sepsis. Therefore, enhancing awareness of the new Sepsis-3 classification system is important, as it may profoundly affect clinicians and their patients.

In the present study, the prognostic performance (crude AUROC) of qSOFA, SIRS and SOFA for predicting adverse outcomes (in-hospital mortality or length of ICU stay $>3$ days) were 0.615 (95\% CI, 0.516-0.707), 0.625 (95\% CI, $0.526-0.716)$ and 0.860 (95\% CI, 0.780-0.920), respectively. Following adjustment for patient age and comorbidities, the values increased to 0.713 (95\% CI, 0.618-0.797), 0.722 (95\% CI, 0.627-0.804) and 0.940 (95\% CI, 0.877-0.977) for qSOFA, SIRS and SOFA, respectively. The results of the assessment of prognostic accuracy were similar to those described in recent studies $(17,19)$. Due to the lack of accurate baseline risk models for in-hospital mortality or ICU stay of $>3$ days, the present study attempted to adjust the model according to several common clinical criteria, including age and comorbidities. In addition, the crude and adjusted AUROC values of SOFA (0.860 and 0.940, respectively) were slightly increased compared with other previous studies (14-17). This may be due to the small and specific study population of the present study or limitations of the study itself. Compared with qSOFA and SIRS, SOFA exhibited a significantly higher sensitivity and specificity. Although the complexity of SOFA scores may 
Table II. Crude and adjusted AUROC.

\begin{tabular}{lccr}
\hline AUROC & qSOFA & SOFA & SIRS \\
\hline Crude AUROC, 95\% CI & $0.615(0.516-0.707)$ & $0.860(0.780-0.920)$ & $0.625(0.526-0.716)$ \\
Adjusted AUROC, 95\% CI & $0.713(0.618-0.797)$ & $0.940(0.877-0.977)$ & $0.722(0.627-0.804)$
\end{tabular}

AUROC, area under the receiver operating characteristic curve; SOFA, sequential (sepsis-related) organ failure assessment; qSOFA, quick sequential (sepsis-related) organ failure assessment; SIRS, systemic inflammatory response syndrome; CI, confidence interval.

Table III. Comparison between crude AUROC.

\begin{tabular}{|c|c|c|c|c|c|}
\hline Comparisons & Difference between areas & $95 \%$ confidence interval & Standard error & Z statistic & P-value \\
\hline qSOFA vs. SIRS & 0.00996 & $-0.128-0.148$ & 0.0703 & 0.142 & 0.8874 \\
\hline qSOFA vs. SOFA & 0.246 & $0.117-0.374$ & 0.0657 & 3.740 & 0.0002 \\
\hline SIRS vs. SOFA & 0.236 & $0.133-0.338$ & 0.0523 & 4.506 & $<0.0001$ \\
\hline
\end{tabular}

SOFA, sequential (sepsis-related) organ failure assessment; qSOFA, quick sequential (sepsis-related) organ failure assessment; SIRS, systemic inflammatory response syndrome.

Table IV. Sensitivity, specificity and likelihood ratios.

\begin{tabular}{lcccccc}
\hline & $\begin{array}{c}\text { Sensitivity, } \% \\
(95 \% \mathrm{CI})\end{array}$ & $\begin{array}{c}\text { Specificity, } \% \\
(95 \% \mathrm{CI})\end{array}$ & $\begin{array}{c}\text { Positive } \\
\text { Predictive value, } \\
\text { Criteria }\end{array}$ & $\begin{array}{c}\text { (95\% CI }) \\
\text { Predictive value, } \\
\%(95 \% \mathrm{CI})\end{array}$ & $\begin{array}{c}\text { Likelihood ratio, } \\
\%(95 \% \mathrm{CI})\end{array}$ & $\begin{array}{c}\text { Likelihood ratio, } \% \\
(95 \% \mathrm{CI})\end{array}$ \\
\hline qSOFA $\geq 2$ & $89.61(80.6-95.4)$ & $26.67(12.3-45.9)$ & $75.8(71.4-79.8)$ & $50.0(29.2-70.8)$ & $1.22(1.0-1.5)$ & $0.39(0.2-0.9)$ \\
qSOFA $\geq 2$ & $97.40(90.9-99.7)$ & $63.33(43.9-80.1)$ & $87.2(81.0-91.6)$ & $90.5(70.2-97.5)$ & $2.66(1.7-4.3)$ & $0.041(0.01-0.2)$ \\
SIRS $>2$ & $93.51(85.5-97.9)$ & $6.67(0.8-22.1)$ & $72.0(69.7-74.2)$ & $28.6(7.6-66.1)$ & $1.00(0.9-1.1)$ & $0.97(0.2-4.8)$ \\
\hline
\end{tabular}

CI, confidence interval; SOFA, sequential (sepsis-related) organ failure assessment; qSOFA, quick sequential (sepsis-related) organ failure assessment; SIRS, systemic inflammatory response syndrome.

hinder its utilization, it remains an accurate and efficient tool for prognostic prediction within or outside the ICU and ED. The sensitivity and specificity values of the SOFA score were sufficient for the clinical application. In addition, no significant difference in the effectiveness of SIRS and qSOFA criteria to predict prognosis among patients with urolithiasis-associated sepsis was observed.

The validity of SIRS criteria as a prognostic tool has been widely challenged. In addition, its poor sensitivity as diagnostic criteria has been criticized for a number of years. SIRS criteria may unable to predict organ dysfunction with mortality in ED patients with suspected infections (25). By contrast, the application of SIRS criteria was associated with an increased 30-day mortality rate among patients who had blood cultures performed from the ED (26). However, the new definition of Sepsis-3 has abandoned this calibration of SIRS criteria and there is greater emphasis on scoring organ dysfunction (1). In the present study, the prognostic accuracies between SIRS and qSOFA were equivalent. Although there have been certain studies that compared the prognostic effectiveness between SIRS and qSOFA, the results are controversial and contradictory $(14,15,17,27,28)$. It is possible that neither are superior for predicting the prognosis of patients with suspected sepsis.

The present study focused on prognoses in patients with suspected urolithiasis-associated sepsis following surgical interventions. Patients with suspected urosepsis but without surgical interventions, such as emergency decompression for obstructive urolithiasis, may exhibit significantly different pathophysiology and outcome. In addition, based on evidence from an American nationwide in-patient study, urgent decompression has been directly associated with a decrease in mortality in patients with sepsis and ureteral calculi (29). In terms of surgery, a number of studies have reviewed the risk factors for the occurrence of postoperative sepsis and mortality following endourological treatment: Presence of kidney stones; large stone burden; female sex; diabetes mellitus; renal insufficiency; high pressure in the collecting system; and long surgery times were identified to be risk factors for sepsis following operation $(9-11,30)$. Wu et al (12) demonstrated an association between the early marked decrease in WBC count and uroseptic shock induced 
Table V. Comparison between adjusted AUROC.

\begin{tabular}{|c|c|c|c|c|c|}
\hline Comparisons & Difference between areas & $95 \%$ confidence interval & Standard error & Z statistic & P-value \\
\hline qSOFA vs. SIRS & 0.00844 & $-0.102-0.119$ & 0.0564 & 0.150 & 0.8811 \\
\hline qSOFA vs. SOFA & 0.227 & $0.134-0.319$ & 0.0473 & 4.800 & $<0.0001$ \\
\hline SIRS vs. SOFA & 0.218 & $0.118-0.319$ & 0.0513 & 4.254 & $<0.0001$ \\
\hline
\end{tabular}

SOFA, sequential (sepsis-related) organ failure assessment; qSOFA, quick sequential (sepsis-related) organ failure assessment; SIRS, systemic inflammatory response syndrome.

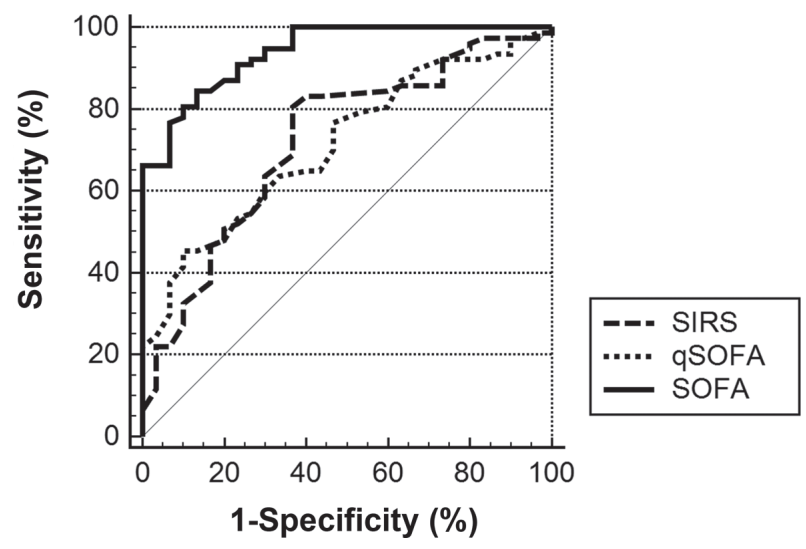

Figure 4. Comparison of prognostic performances with adjusted area under the receiver operating characteristic curve values. SOFA, sequential (sepsis-related) organ failure assessment; SIRS, systemic inflammatory response syndrome; qSOFA, quick sequential (sepsis-related) organ failure assessment.

by upper urinary tract endoscopic lithotripsy. However, there was no unified, widely accepted and standardized criteria for predicting the prognosis of these patients. Therefore, the prognostic accuracy of the generic criteria for these specific patients was validated in the present study. The results demonstrated that the SOFA score was a preferable prognostic tool for patients with urolithiasis-associated sepsis following surgical interventions. Prognostic performances of SOFA were validated by a large retrospective cohort analysis of adult patients with suspected infection in Australia and New Zealand (15). Among 184,875 patients, SOFA exhibited significantly greater prognostic accuracy for adverse outcome (in-hospital mortality) compared with SIRS criteria or qSOFA scores, with a crude AUROC value of 0.753 (15). However, in a second international prospective cohort study, among patients admitted to the ED with suspected infection, the use of qSOFA exhibited greater prognostic accuracy for mortality (in-hospital) compared with Sepsis-1 criteria (SIRS and severe sepsis) (14). These results indicated that the Sepsis-3 criteria (SOFA and qSOFA) should be recommended in the ED setting. The results of the present study suggest that urologists should consider the SOFA score of patients with urolithiasis-associated sepsis when considering prognoses. The prognostic performance of SOFA for in-hospital mortality or prolonged ICU stay (>3 days) among patients with urolithiasis-associated sepsis transferred to ICU following surgical interventions was significantly increased compared with those of qSOFA and SIRS.

The present study had a number of limitations. Firstly, it was a single-center retrospective study with unavoidable selection bias. In addition, due to the limited clinical data, only the patients transferred to SICU following surgical interventions were included in the analysis, in spite of routine institutional (Xin Hua Hospital Affiliated to Shanghai Jiao Tong University) regulations, which state that all patients with suspected sepsis in surgical departments should be transferred to SICU. The decision for transfer to SICU is generally made by the primary surgeon of the patient or the senior on-duty surgeon, and it may have been affected by previous experiences of the clinicians. In addition, the diagnostic criteria of sepsis were changed following the proposal of Sepsis-3, a new diagnostic definition of sepsis, in 2016 (3). In addition, different types of surgical interventions may lead to different outcomes; therefore, the sample patient population was not homogeneous. However, Sepsis-3 and SIRS criteria are generic diagnostic and prognostic tools used for different types of sepsis, derived from different systems. Therefore, the heterogeneity of patients observed in the present study was considered to be acceptable, however, it should be taken into consideration as it may have affected the results. In addition, the study population size was small; therefore, the outcome may be inaccurate and affected by random error. To validate the results of the present study, a multi-center prospective study is required.

\section{Acknowledgements}

Not applicable.

\section{Funding}

The present study was funded by Shanghai Municipal Commission of Health and Family Planning Grant (grant no. 201440314), Shanghai Municipal Commission of Science and Technology Grant (grant no. 17441905602), National Natural Science Fund for Young Scholars (grant no. 81802522) and Shanghai Sailing Program (grant no. 18YF1415200).

\section{Availability of data and materials}

The datasets used and/or analyzed during the present study are available from the corresponding author on reasonable request. 


\section{Authors' contributions}

YH and JD supervised the project. JD designed the study. BS, FS, KX, LS and JG collected the data. BS, HT, NW and YW performed statistical analyses. YH performed surgical procedures. BS, JD and FS wrote the manuscript. YW and JD corrected the language of manuscript.

\section{Ethics approval and consent to participate}

Written informed consent was provided by each patient prior to surgery. The Ethics Committee of Xin Hua Hospital Affiliated to Shanghai Jiao Tong University School of Medicine approved the retrospective study and permitted the waiver of written informed consent from patients (approval no., XHEC-D-2018-074). The study protocol was also in accordance with the Declaration of Helsinki.

\section{Patient consent for publication}

Not applicable.

\section{Competing interests}

The authors declare that they have no competing interests.

\section{References}

1. Gotts JE and Matthay MA: Sepsis: Pathophysiology and clinical management. BMJ 353: i1585, 2016.

2. Cohen J, Vincent JL, Adhikari NK, Machado FR, Angus DC, Calandra T, Jaton K, Giulieri S, Delaloye J, Opal S, et al: Sepsis: A roadmap for future research. Lancet Infect Dis 15: 581-614, 2015.

3. Singer M, Deutschman CS, Seymour CW, Shankar-Hari M, Annane D, Bauer M, Bellomo R, Bernard GR, Chiche JD, Coopersmith CM, et al: The third international consensus definitions for sepsis and septic shock (Sepsis-3). JAMA 315: 801-810, 2016.

4. Shankar-Hari M, Phillips GS, Levy ML, Seymour CW, Liu VX, Deutschman CS, Angus DC, Rubenfeld GD and Singer M; Sepsis Definitions Task Force: Developing a new definition and assessing new clinical criteria for septic shock: For the third international consensus definitions for sepsis and septic shock (Sepsis-3). JAMA 315: 775-787, 2016.

5. Seymour CW, Liu VX, Iwashyna TJ, Brunkhorst FM, Rea TD, Scherag A, Rubenfeld G, Kahn JM, Shankar-Hari M, Singer M, et al: Assessment of clinical criteria for sepsis: For the third international consensus definitions for sepsis and septic shock (Sepsis-3). JAMA 315: 762-774, 2016.

6. Dreger NM, Degener S, Ahmad-Nejad P, Wöbker G and Roth S: Urosepsis-etiology, diagnosis and treatment. Dtsch Arztebl Int 112: 837-848, 2015.

7. Wagenlehner FM, Pilatz A, Weidner W and Naber KG: Urosepsis: Overview of the diagnostic and treatment challenges. Microbiol Spectr: 3, 2015 doi: 10.1128/microbiolspec.UTI-0003-2012.

8. Wagenlehner FM, Lichtenstern C, Rolfes C, Mayer K, Uhle F, Weidner W and Weigand MA: Diagnosis and management for urosepsis. Int J Urol 20: 963-970, 2013.

9. Michel MS, Trojan L and Rassweiler JJ: Complications in percutaneous nephrolithotomy. Eur Urol 51: 899-906, 2007.

10. Koras O,Bozkurt IH, Yonguc T, Degirmenci T, Arslan B, Gunlusoy B, Aydogdu $\mathrm{O}$ and Minareci S: Risk factors for postoperative infectious complications following percutaneous nephrolithotomy: A prospective clinical study. Urolithiasis 43: 55-60, 2015.

11. Çıtamak B, Altan M, Bozacı AC, Koni A, Doğan HS, Bilen CY, Şahin A and Tekgül S: Percutaneous nephrolithotomy in children: 17 Years of experience. J Urol 195: 1082-1087, 2016.

12. Wu H, Zhu S, Yu S, Ding G, Xu J, Li T, Qiao L, Chen Y, Yan J, Cheng X, et al: Early drastic decrease in white blood count can predict uroseptic shock induced by upper urinary tract endoscopic lithotripsy: A translational study. J Urol 193: 2116-2122, 2015.
13. Hu M, Zhong X, Cui X, Xu X, Zhang Z, Guan L, Feng Q, Huang Y and $\mathrm{Hu}$ W: Development and validation of a risk-prediction nomogram for patients with ureteral calculi associated with urosepsis: A retrospective analysis. PLoS One 13: e0201515, 2018.

14. Freund Y, Lemachatti N, Krastinova E, Van Laer M, Claessens YE, Avondo A, Occelli C, Feral-Pierssens AL, Truchot J, Ortega M, et al: Prognostic accuracy of sepsis-3 criteria for in-hospital mortality among patients with suspected infection presenting to the emergency department. JAMA 317: 301-308, 2017.

15. Raith EP, Udy AA, Bailey M, McGloughlin S, MacIsaac C, Bellomo R and Pilcher DV; Australian and New Zealand Intensive Care Society (ANZICS) Centre for Outcomes and Resource Evaluation (CORE): Prognostic accuracy of the SOFA score, SIRS criteria and qSOFA score for in-hospital mortality among adults with suspected infection admitted to the intensive care unit. JAMA 317: 290-300, 2017.

16. Tokioka F, Okamoto H, Yamazaki A, Itou A and Ishida T: The prognostic performance of qSOFA for community-acquired pneumonia. J Intensive Care 6: 46, 2018.

17. Singer AJ, Ng J, Thode HC Jr, Spiegel R and Weingart S: Quick SOFA scores predict mortality in adult emergency department patients with and without suspected infection. Ann Emerg Med 69: 475-479, 2017.

18. Park HK, Kim WY, Kim MC, Jung W and Ko BS: Quick sequential organ failure assessment compared to systemic inflammatory response syndrome for predicting sepsis in emergency department. J Crit Care 42: 12-17, 2017.

19. Williams JM, Greenslade JH, McKenzie JV, Chu K, Brown AFT and Lipman J: Systemic inflammatory response syndrome, quick sequential organ function assessment, and organ dysfunction: Insights from a prospective database of ED patients with infection. Chest 151: 586-596, 2017.

20. Luo X, Yang X, Li J, Zou G, Lin Y, Qing G, Yang R, Yao W and Ye X: The procalcitonin/albumin ratio as an early diagnostic predictor in discriminating urosepsis from patients with febrile urinary tract infection. Medicine (Baltimore) 97: e11078, 2018.

21. Wu H, Wang Z, Zhu S, Rao D, Hu L, Qiao L, Chen Y, Yan J, Chen X, Wan SP, et al: Uroseptic shock can be reversed by early intervention based on leukocyte count $2 \mathrm{~h}$ post-operation: Animal model and multicenter clinical cohort study. Inflammation 41: 1835-1841, 2018.

22. Wagenlehner FM, Tandogdu Z and Bjerklund Johansen TE: An update on classification and management of urosepsis. Curr Opin Urol 27: 133-137, 2017.

23. Türk C, Petř́́k A, Sarica K, Seitz C, Skolarikos A, Straub M and Knoll T: EAU guidelines on interventional treatment for urolithiasis. Eur Urol 69: 475-482, 2016.

24. Rhodes A, Evans LE, Alhazzani W, Levy MM, Antonelli M, Ferrer R, Kumar A, Sevransky JE, Sprung CL, Nunnally ME, et al: Surviving sepsis campaign: International guidelines for management of sepsis and septic shock: 2016. Crit Care Med 45: 486-552, 2017.

25. Shapiro N, Howell MD, Bates DW, Angus DC, Ngo L and Talmor D: The association of sepsis syndrome and organ dysfunction with mortality in emergency department patients with suspected infection. Ann Emerg Med 48: 583-590.e1, 2006.

26. Lindvig KP, Nielsen SL, Henriksen DP, Jensen TG, Kolmos HJ, Pedersen C, Vinholt PJ and Lassen AT: Mortality and prognostic factors of patients who have blood cultures performed in the emergency department: A cohort study. Eur J Emerg Med 23: 166-172, 2016.

27. Finkelsztein EJ, Jones DS, Ma KC, Pabón MA, Delgado T, Nakahira K, Arbo JE, Berlin DA, Schenck EJ, Choi AM and Siempos II: Comparison of qSOFA and SIRS for predicting adverse outcomes of patients with suspicion of sepsis outside the intensive care unit. Crit Care 21: 73, 2017.

28. Ho KM and Lan NS: Combining quick sequential organ failure assessment with plasma lactate concentration is comparable to standard sequential organ failure assessment score in predicting mortality of patients with and without suspected infection. J Crit Care 38: 1-5, 2017.

29. Borofsky MS, Walter D, Shah O, Goldfarb DS, Mues AC and Makarov DV: Surgical decompression is associated with decreased mortality in patients with sepsis and ureteral calculi. J Urol 189: 946-951, 2013.

30. de la Rosette J, Denstedt J, Geavlete P, Keeley F, Matsuda T, Pearle M, Preminger G and Traxer O; CROES URS Study Group: The clinical research office of the endourological society ureteroscopy global study: Indications, complications, and outcomes in 11,885 patients. J Endourol 28: 131-139, 2014. 